\title{
Investigating environmental trends in the outer visible edges of dwarf irregular galaxies
}

\author{
Jacqueline M. Dunn \\ Midwestern State University \\ 3410 Taft Blvd., Wichita Falls, TX 76308, USA \\ email: jackie.dunn@mwsu.edu
}

\begin{abstract}
The star formation histories and evolution of 28 dwarf irregular galaxies (dIs) that reside in differing local and global environments are investigated. The shallow gravitational potentials of dwarf galaxies make these objects highly susceptible to changes in morphology or dynamics by external perturbations. Additionally, the lack of more complicated structures such as spiral arms makes environmental effects more easily discernible. Therefore, dIs are ideal candidates for a study of the role of environment in galaxy evolution. The local environment is defined by the local galaxy number density, where high indicates at least one neighbor within $200 \mathrm{kpc}$ and low indicates no neighbors within $1 \mathrm{Mpc}$. The global environment is classified as either the field or a galaxy group / cluster.

Absolute magnitudes, colors, central surface brightnesses, star formation rates and color profiles were compared using photometry from UBVR and $\mathrm{H} \alpha$ imaging. While some environmental trends are noted (galaxies in local high density environments have brighter central and effective surface brightnesses, while those in global high density environments have brighter absolute magnitudes, central and effective surface brightnesses, and higher star formation rates), no systematic environmental trends are seen in the shape of the color profiles or spatial distribution of recent star formation. A lack of environmental trend in star formation and galaxy color indicates that either internal processes dominate the formation and subsequent evolution of the outskirts of dIs, or there is no systematic environmental effect on such. The appearance of environmental effects should be more noticeable at the outermost edges of these systems. The lack of a trend in such implies that the exact nature of the environmental influence varies greatly depending on the exact nature of the interaction occurring.
\end{abstract}

Keywords. galaxies: dwarf, galaxies: irregular

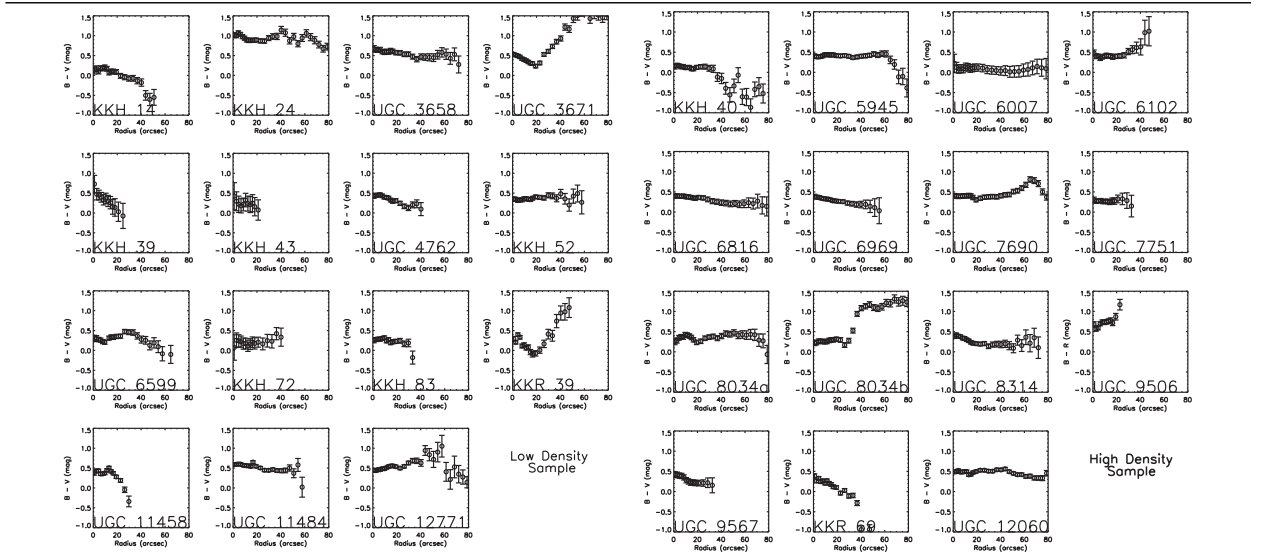

Figure 1. B-V color profiles (B-V ranges from -1.0 to $1.5 \mathrm{mag}$, radius 0 to 80 arcseconds). Note the shape of the profile does not depend on environment (low density sample on left, high density sample on right). 